\title{
Analysis of Ionizing Radiation Generated by Partial Discharges
}

\author{
D. ZMARZEY*, E. NAGI, S. BorUCKi AND T. BoCZAR \\ Faculty of Electrical Engineering, Automatic Control and Computer Science, Opole University of Technology \\ Prószkowska 76, 45-758 Opole, Poland
}

This paper presents the preliminary results of the performed experiment, based on common phenomenon of partial discharges. A simple Geiger-Muller counter (DP-66M) was used for measurement of a dose of ionizing radiation, which is accompanied by partial discharges. Values from which the radiation intensity was dependent could be controlled individually: the distance between measurer and source, as well as the voltage generating partial discharges. The obtained results indicate that in the examined phenomenon, significant dose of X-ray radiation is present. According to the Kramers-Kulenkampff theory, it depends also on the atomic number of elements involved. Using MATLAB software, the obtained data were compiled to develop suitable theory for further research study. There was also implemented fuzzification for the fuzzy logic, and the effect was the capability of forecasting radiation doses according to the fixed variables for a given material, where partial discharges were generated.

DOI: 10.12693/APhysPolA.125.1377

PACS: 43.40.-r, 43.40.Vn, 43.58.Wc

\section{Introduction}

In insulations of electrical devices and cables, local breakdowns can occur, named partial discharges (PDs). Their generation and development affect a number of physical phenomena, among others, including: current pulse and emission of electromagnetic wave, light radiation, local temperature rise in the discharge area, and gas pressure change in its channel, etc. These effects can occur with varying intensity, and it is significantly influenced by the type of dielectric insulation system used, and the type of PDs. Based on observations of the aforementioned phenomena, many methods for their detection, measurement and location were developed [1-4]. In recent times, non-destructive testing methods have gained a greater importance. They allow performing insulation condition assessment during operation of power equipment $[5,6]$. Research studies on ionizing radiation may become a new non-invasive method to assess the condition of insulation.

Ionizing radiation is a common and important phenomenon in the surrounding world. Types of ionizing radiation have impact on effects that arise due to interference, and defects in the structure of a given material and changes in the elemental composition are directly derived from radioactive changes. There are four types of ionizing radiation: alpha radiation (helium nuclei), beta radiation (electrons or positrons), neutron radiation, and electromagnetic radiation (X-ray, braking, and gamma radiation). One of the factors related to the type of radiation and its energy is permeability. The lowest permeability has alpha radiation (a few sheets of paper is

\footnotetext{
*corresponding author; e-mail: dzmarzly@gmail.com
}

capable to secure the matter from helium nuclei), while the highest one is characterized by electromagnetic radiation $[7,8]$.

\section{Ionizing radiation resulting from PDs}

During PDs formed in cable insulation (polyolyphine) there was observed X-rays occurrence. That component of radiation is able to infiltrate through the thin layer of light metal, such as beryllium or aluminium, and thus it would be possible to detect PDs in this way. It is possible to take X-ray image of voids in insulation of electric cables, and this would enable non-invasive assessment of losses and insulation damages. It has been previously shown that X-ray component of partial discharges is so called braking radiation, and intensity of X-ray partial discharges is dependent on number of gases atoms present in the voids where PD takes place. In addition, the atomic number $Z$ of the elements in the gas mixture generates dependence consistent with the KramersKulenkampff theory [9]. According to the formula

$$
P=\frac{C\left[Z\left(v-v_{0}\right)+b Z^{4}\right]}{R^{2}} .
$$

Another type of radiation observed in the PDs generation is a beta radiation. Indication was made with X-ray radiometer DP-66M with STS-5 detectors (Fig. 1).

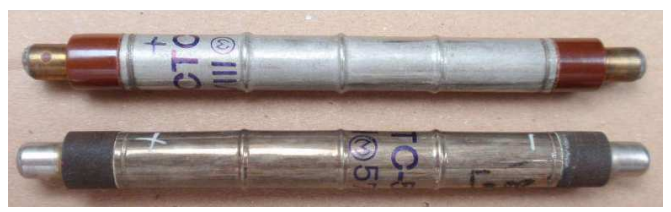

Fig. 1. Geiger counter STS-5 detector used in X-ray radiometer DP-66M. 


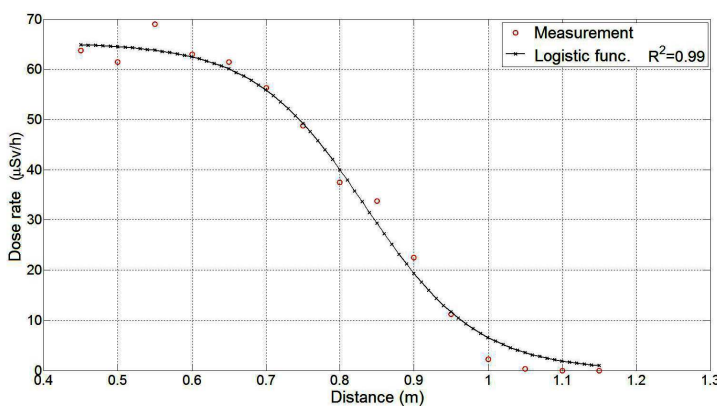

Fig. 2. Dependence of the dose rate of radiation as a function of distance from the PD source $P=f(r)$.

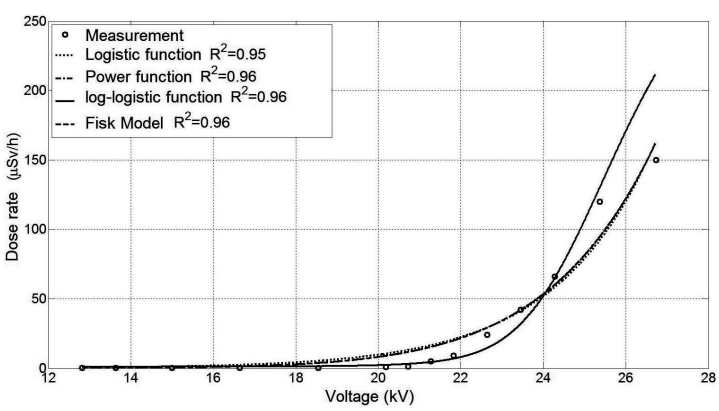

Fig. 3. Dependence of the dose rate of radiation as a function of voltage causing $\mathrm{PD} P=f(U)$.

There was tested a dependence between radiation dose resulting from $\mathrm{PDs}$ and voltage causing discharges and in dependence of probe distance from source of PDs. Preliminary results showed a large proportion of the dose rate of radiation both in relation to the distance from the source of partial discharges and the voltage causing it. Dependences are shown in Figs. 2 and 3. The medium where radiation beta was depressed was air.

\section{Theoretical prediction}

For effective phenomenon research there should be created the proper theory and tools to predict doses obtained. Efficacy and safety of the experiment could be obtained with the help of MATLAB environment. In the Fuzzy Logic toolbox there was made a fuzzification of data, which we obtained in preliminary experiments, in order to develop efficient tool for result prediction in dependence on the initial conditions. The developed tool effectively draws results, as it was shown in Fig. 4.

To make fuzzification work correctly, in the first place, functions of membership were determined for sets of input data: $X_{1}$ - voltage causing PDs, $X_{2}$ - meter distance from the source of PD (fixed).

Membership functions have been defined on the basis of the initial graph (Fig. 5) obtained from experimental data. To solve the task there was used MATLAB environment, wherein the Mamdani model was implemented. Conclusion process in MATLAB is automatic.

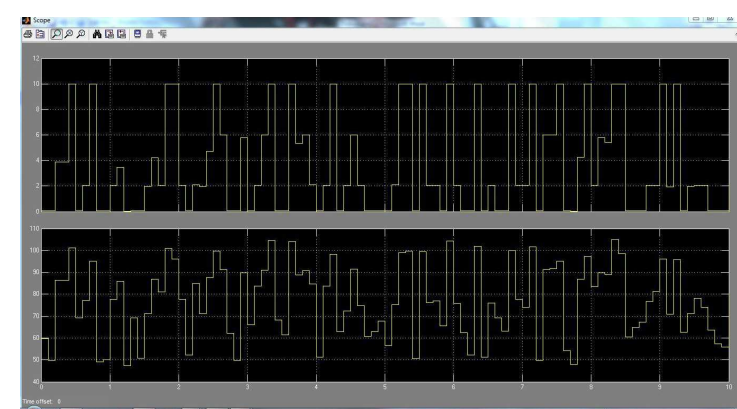

Fig. 4. Graphical representation of the prediction of the output data after fuzzification.

Rules databases used in the process of fuzzification have been determined assuming that one set of input data has constant values.

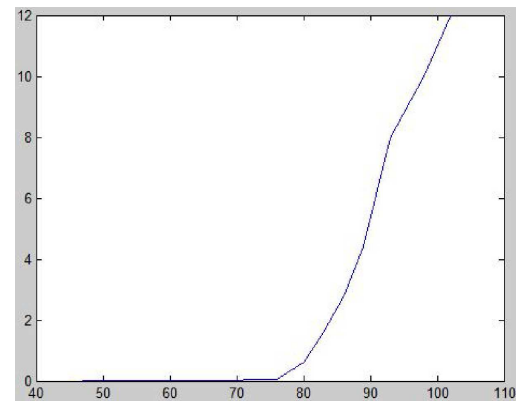

Fig. 5. Initial graph from experimental data.

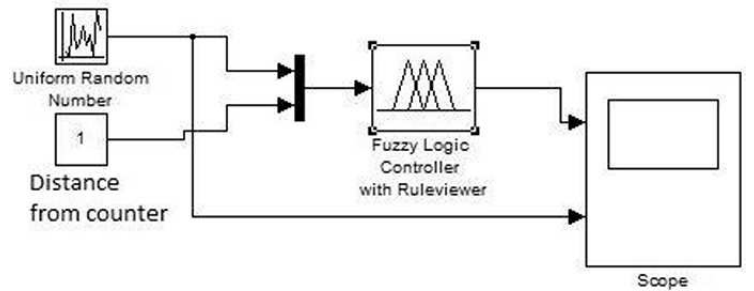

Fig. 6. Fuzziness mechanism.

Applied fuzziness scheme used for determining the output parameters of the experiment is shown in Fig. 6 . Membership function for the output data (radiation dose rate) was also determined by the initial graph from experimental data.

\section{Conclusion}

Detection of PDs using radiation measurements can become a new method for diagnostics in electric power devices and networks. Thanks to accurate, and inexpensive hardware based on popular Geiger-Muller counters, there can be determined the radiation power associated with partial discharges, and thus the size of damage of electrical insulation. Accurate development of the 
method allows for a quick and relatively inexpensive test of insulation condition of many difficult to access equipment and power lines. The new method would have a substantial impact on the work of people involved in the diagnosis, directly in terms of health and safety. In times of planned energy investments in Poland, as nuclear power plants, it could become one of the major methods. This method can also reduce the cost of security at nuclear power plants by using the same basic measuring instruments for the detection of ionizing radiation from radioactive decay.

\section{References}

[1] A. Cichoń, Acta Phys. Pol. A 116, 290 (2009).

[2] D. Wotzka, T. Boczar, P. Fracz, Acta Phys. Pol. A 120, 767 (2011).
[3] S. Borucki, Acta Phys. Pol. A 120, 571 (2011).

[4] D. Wotzka, D. Zmarzly, T. Boczar, Acta Phys. Pol. A 118, 1272 (2010).

[5] D. Wotzka, T. Boczar, Przeglad Elektrotechniczny 11b, 74 (2010) (in Polish).

[6] P. Frącz, Measurments Automatics Control 1, 50 (2009) (in Polish).

[7] K.N. Muchin, Experimental Nuclear Physics. Physics of Atomic Nucleus, WNT, Warsaw 1978 (in Polish).

[8] B. Dziunikowski, On Physics and Nuclear Power, AGH, Kraków 2001, (in Polish).

[9] G.K. Novikov, A.I. Smirnov, V.V. Fedchiskin, Russ. Electr. Eng. 80, 617 (2009). 